\title{
Nectar Intake of White-Bellied Sunbirds (Cinnyris talatala): Can Meal Size Be Inferred from Feeding Duration?
}

\author{
Angela Köhler ${ }^{*}$ \\ Luke Verburgt \\ Sue W. Nicolson \\ Department of Zoology and Entomology, University of \\ Pretoria, Pretoria 0002, South Africa \\ Accepted 2/27/2008; Electronically Published 8/26/2008
}

\begin{abstract}
A positive relationship between feeding duration and meal size of avian nectarivores has often been assumed in earlier studies. We investigated whether feeding duration can be used as a surrogate for the amount of sugar solution ingested by whitebellied sunbirds, Cinnyris (Nectarinia) talatala. Feeding durations of sunbirds consuming three sucrose concentrations $(10 \%, 20 \%$, and $40 \% \mathrm{w} / \mathrm{w})$ were measured using an infrared photodetection system, and the amounts consumed were recorded simultaneously by weighing the feeder throughout the experiment. For all three diet concentrations, a positive relationship was found between the time spent feeding per $30 \mathrm{~min}$ and the mass consumed. Therefore, feeding duration is demonstrated to be an index of the amount ingested on a particular sugar concentration. The rate of ingestion, however, depended on the sugar concentration, with the highest rate at the lowest concentration of $10 \%$ and the lowest rate at the $40 \%$ concentration. Less total time was spent feeding on the $20 \%$ solution than on the $10 \%$ solution, but time increased on the $40 \%$ diet because of viscosity effects. There appeared to be a weak relationship between feeding patterns and sex, but this was not significant, probably because of interindividual variation.
\end{abstract}

\section{Introduction}

Food intake as a fundamental physiological function of animals has been extensively investigated. Feeding patterns are examined by measuring characteristic parameters such as meal size and feeding duration. Meal size can be recorded by weighing

*Corresponding author; e-mail: akoehler@zoology.up.ac.za.

Physiological and Biochemical Zoology 81(5):682-687. 2008. (C) 2008 by The University of Chicago. All rights reserved. 1522-2152/2008/8105-8027\$15.00 DOI: $10.1086 / 588174$ the food continuously, for example, by placing food containers on scales as in experiments with rats (Blanton et al. 1998; Kahler et al. 1998). In nectar-feeding insects, meal size has been measured by weighing the insect before and after the meal (Hainsworth et al. 1991; Paul and Roces 2003). For avian nectarivores, however, food intake is commonly determined only hourly or daily. Nectar intake has been measured by weighing the feeder (Nicolson and Fleming 2003; Nicolson et al. 2005) or by reading changes in fluid meniscus levels in glass or plastic feeders, as done for honeyeaters (Collins and Clow 1978), sunbirds and sugarbirds (Jackson et al. 1998), and hummingbirds (LópezCalleja and Bozinovic 2003). Feeding durations of nectar-feeding birds, however, have been recorded with a stopwatch (Collins and Clow 1978) or a video camera (Mitchell and Paton 1990). Feeding durations have also been measured using a photodetection system and timer, where a photo beam is broken when the bird feeds (Gass 1978; Garrison and Gass 1999; Köhler et al. 2006).

The relationship between feeding duration and meal size has often been assumed in previous studies on nectar-feeding birds. Mitchell and Paton (1990), for instance, recorded honeyeaters' handling times for artificial flowers containing nectar of known volume and concentration. They calculated sugar intake rate, assuming that handling time is positively related to the volume consumed. However, this relationship has not been sufficiently investigated in avian nectarivores. To our knowledge, only one study, on western spinebills (Acanthorhynchus superciliosis), has demonstrated that longer feeding durations are reflected in larger volumes of nectar ingested (Collins and Clow 1978).

Previously we used an infrared photodetection system to investigate short-term feeding patterns of white-bellied sunbirds, Cinnyris (Nectarinia) talatala, fed on artificial nectar of various sugar concentrations (Köhler et al. 2006). However, the relationship between feeding duration of the sunbirds and their meal size could not be determined directly. We used feeding duration as an estimate of meal size, based on the assumption that intake rates should be constant for birds feeding on a single sugar concentration. This study aimed to determine whether feeding duration of white-bellied sunbirds can be used as a surrogate for the amount of sugar solution ingested. Here we defined meal size as the amount of sugar solution ingested in $30 \mathrm{~min}$ because the minute amounts of solution ingested in a single feeding event could not be detected. We also compared males and females to determine whether there is a sex-specific feeding pattern in this species. 


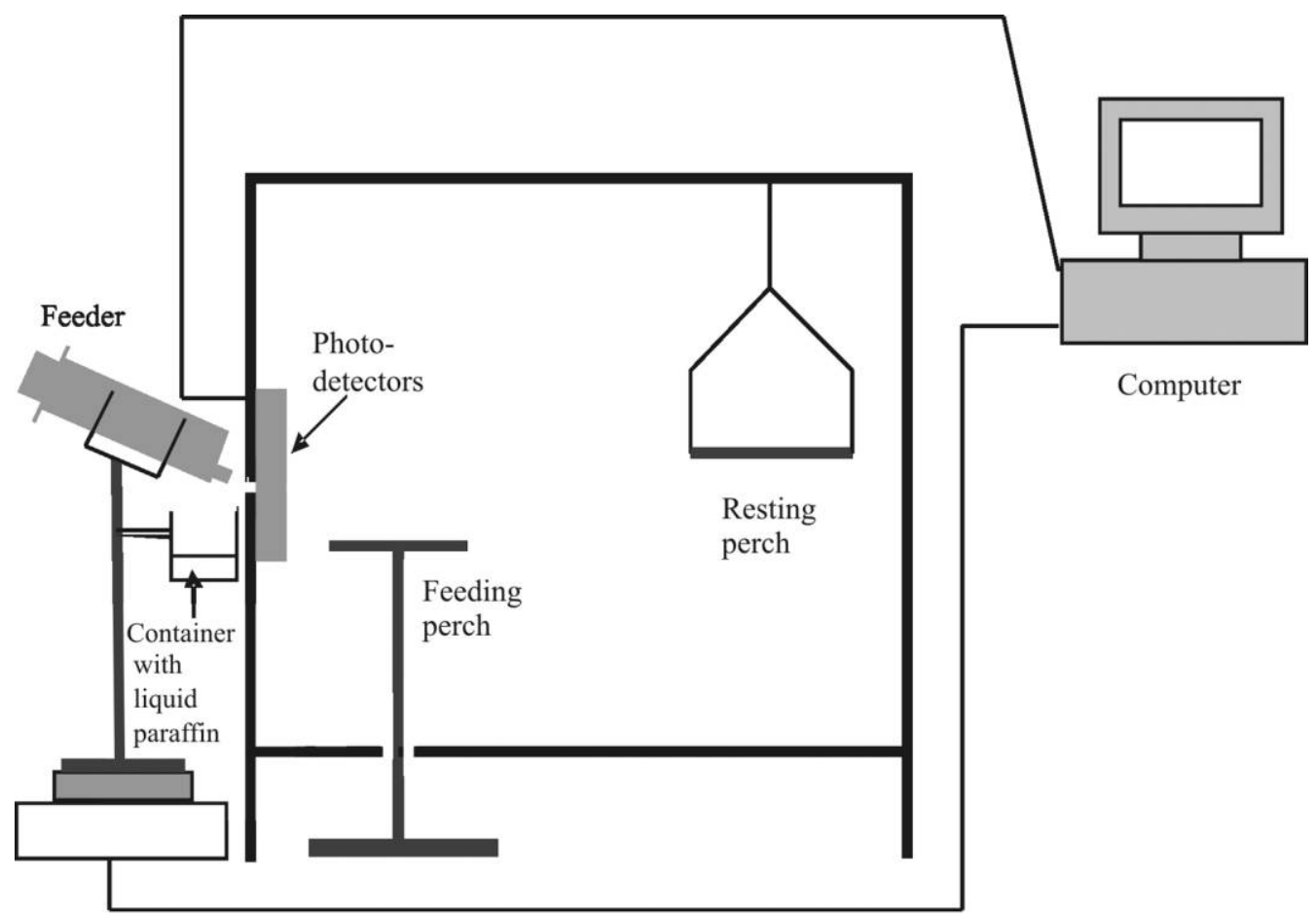

Electronic balance

Figure 1. The experimental cage was constructed from Perspex and contained two perches. An infrared photodetection system, interfaced to a computer, was set up on either side of the feeding aperture to record feeding events. Next to the cage, the feeder with sugar solution was mounted on an electronic balance, which was interfaced to the same computer.

\section{Material and Methods}

\section{Study Animals and Their Maintenance}

Ten nonreproductive white-bellied sunbirds were captured with mist nets in Jan Cilliers Park, Pretoria. Birds were initially housed together in an outdoor aviary measuring $8 \mathrm{~m} \times 5$ $\mathrm{m} \times 2 \mathrm{~m}$. One week before the experiments, birds were moved to individual cages of $45 \mathrm{~cm} \times 45 \mathrm{~cm} \times 32 \mathrm{~cm}$ in a climatecontrolled room. They were acclimated to the experimental temperature of $20^{\circ} \pm 2^{\circ} \mathrm{C}$ and a $12 \mathrm{~L}: 12 \mathrm{D}$ photoperiod, with lights on at 0700 hours. The maintenance diet consisted of $20 \%$ w/w sucrose $(0.63 \mathrm{M})$ with a nutritional supplement (Ensure, Abbott Laboratories, Johannesburg, South Africa) to provide dietary nitrogen (van Tets and Nicolson 2000). The maintenance diet and supplementary water were provided ad lib. in inverted, stoppered syringes. Body mass (mean \pm SE) of five males and five females was $9.31 \pm 0.39 \mathrm{~g}$ and $8.08 \pm 0.18 \mathrm{~g}$, respectively. Our experiments were approved by the Animal Use and Care Committee of the University of Pretoria (AUCC 060515-012).

\section{Experimental Design}

Each sunbird received $10 \%, 20 \%$, and $40 \%$ w/w sucrose solutions $(0.32,0.63$, and $1.35 \mathrm{M})$ in randomized order for a period of $24 \mathrm{~h}$ each. The experimental diet was changed every morning before the lights were turned on. To prevent mass loss on sugar-only diets (Nicolson and Fleming 2003), all experimental diets contained Ensure. The amount of Ensure added was adjusted according to the sugar concentration of the experimental diet because the volumes ingested are inversely proportional to sugar concentration. This means that the $40 \%$ diet contained the largest amount of Ensure to keep protein intake constant. Even if the carbohydrate component of the added Ensure were completely digested by sunbirds, it would increase each sugar concentration by a maximum of only $5.8 \%$, for example, an increase from $10 \% \mathrm{w} / \mathrm{w}$ to $10.58 \% \mathrm{w} / \mathrm{w}$, and is therefore negligible.

Birds were moved sequentially to an experimental cage (Köhler et al. 2006) and were allowed to acclimate for $1 \mathrm{~d}$ before measurements commenced. The cage $(50 \mathrm{~cm} \times 40 \mathrm{~cm} \times 45$ $\mathrm{cm}$ ) was constructed from Perspex with ventilation holes and contained a feeding perch and a resting perch (Fig. 1). Automated recording of the time spent feeding and the number of feeding events was achieved by an infrared photodetection system interfaced to a computer (Köhler et al. 2006). Outside the cage, the feeder with the sucrose solution was mounted on an electronic balance (Mettler Toledo PB-602S, 0.01-g accuracy, Microsep, Johannesburg) that was interfaced to the same computer. Feeder mass was recorded every $0.2 \mathrm{~s}$ to determine the mass consumed by the bird. Occasional drips were collected in a container with liquid paraffin (to avoid evaporative mass loss), 
which was also placed on the electronic balance (Fig. 1). Thus, the dripping solution did not affect the results.

\section{Data Processing}

For each bird, we obtained the start and end times of all feeding events and their durations ( $\pm 0.001 \mathrm{~s}$ ). We defined feeding event duration as the time between insertion of the bill into the sucrose solution and its removal (Köhler et al. 2006). Feeding event durations of less than $0.1 \mathrm{~s}$ were excluded from analysis, and separate feeding events were merged into a single feeding event when the interval between them was less than $0.25 \mathrm{~s}$ (Köhler et al. 2006).

The light period of the day was divided into 24 intervals of $30 \mathrm{~min}$ each. The first and last of these were excluded because individuals differed in the time of their first and last feeding event of the day (described in detail in Köhler et al. 2006), leaving 22 intervals that were used for analysis. For each individual and each diet concentration, the number of feeding events was calculated for each of the 30-min intervals, defined as feeding frequency. Furthermore, the total time that the bird spent feeding during each 30-min interval, defined as feeding duration, was determined by summing the durations of all feeding events. Although we recorded the feeder mass every 0.2 $\mathrm{s}$, the minute amount of sugar solution consumed in a single feeding event could not be detected because the resolution of our balance was too coarse $(0.01 \mathrm{~g})$. Therefore, we defined the mass consumed per 30-min interval as meal size. Meal size was calculated by subtracting the last feeder mass record of each 30-min interval from the first one. Furthermore, we determined the mass of solution consumed daily by summing the meal size of each interval. From this, the daily sucrose intake was calculated for all birds on all diet concentrations.

\section{Statistical Procedures}

Meal Size and Feeding Behavior. Linear regressions were calculated to determine relationships between feeding duration and meal size $(a)$ and feeding frequency and meal size $(b)$ on all diets. For each relationship ( $a$ and $b$ ), data were arranged for each individual $(n=10)$ and for each diet concentration $(n=3)$ in 30 -min intervals $(n=22)$. This meant that each regression ( $n=30$ for each relationship) was based on 22 data points. This analysis was performed for each individual since we demonstrated large differences in feeding pattern between individuals in our previous study (Köhler et al. 2006). The regression slopes obtained from $a$ are a measure of intake rate and are hereafter exclusively referred to as "intake rate." Intake rates of each individual were compared between the three diet concentrations by repeated-measures ( $\mathrm{rm}$ )ANOVA.

Differences between the Sexes and Body Mass Relationships. Data were tested for normality and homogeneity of variance; log transformations were used when data were not normally distributed. Although body mass data were normally distributed
(Kolmogorov-Smirnov test: $D>0.21 ; P>0.20$ ), we used the more conservative nonparametric Mann-Whitney $U$-test to test for differences in body mass between males and females because of the problems associated with distribution estimation from small sample sizes (Zar 1999). We conducted rmANOVAs to determine differences between the sexes in feeding duration, feeding frequency, and meal size. Data were arranged so that intervals $(n=22)$ were nested within each diet concentration $(n=3)$, with sex being the categorical predictor (betweeneffect). The intake rates obtained from $a$ were also subjected to rmANOVA to test for differences between the sexes. Intake rates of the 10 sunbirds on all diet concentrations $(n=3)$ were dependent variables, with sex as the categorical predictor. Linear regressions were calculated to determine sex-independent relationships between body mass and feeding duration over the whole day and between body mass and mass of solution consumed daily for all diets. For each relationship, data were arranged for each diet concentration $(n=3)$, by individual $(n=10)$. This meant that each regression $(n=3$ for each relationship) was based on 10 data points.

Daily Rhythm, Feeding Patterns, and Consumption on the Different Diets. Data were tested for normality and homogeneity of variance; square root transformations were used when data were heteroscedastic. We performed rmANOVA to determine differences in feeding duration and meal size throughout the day (between 30-min periods) and between diet concentrations. Data were arranged as follows: intervals $(n=22)$ were nested within sucrose concentration $(n=3)$, yielding 66 dependent variables. The daily mass of solution consumed and sucrose intake of the 10 sunbirds were compared between the diet concentrations $(n=3)$ by rmANOVA.

Linear regressions describing individual relationships between feeding behavior and meal size were followed by a sequential Bonferroni correction to avoid Type I errors (Rice 1989). Post hoc comparisons for all rmANOVA were conducted with Tukey's honest significant difference test for equal sample sizes and followed by a Bonferroni correction for multiple comparisons. All data are presented as means \pm SE. Before the Bonferroni corrections, the level of significance was $P \leq 0.05$ for all tests.

\section{Results}

Meal Size and Feeding Behavior

Feeding duration. The feeding duration of each sunbird was positively related to meal size for all three sucrose concentrations (all $F_{1,20} \geq 4.54$, all $P \leq 0.05$, all $R^{2} \geq 0.19$ ), except for one individual on the $40 \% \operatorname{diet}\left(F_{1,20}=2.94, P=0.10, R^{2}=0.13\right)$. However, this bird was not excluded from the analysis because there was no obvious reason, such as poor condition of the bird or equipment malfunction, to justify its exclusion. Figure 2 shows the positive relationship between meal size and feeding duration of all 10 birds for each sucrose concentration (10\%: $y=0.013[ \pm 0.0004] x+0.125, \quad R^{2}=0.98 ; \quad 20 \%: \quad y=0.011[ \pm$ 


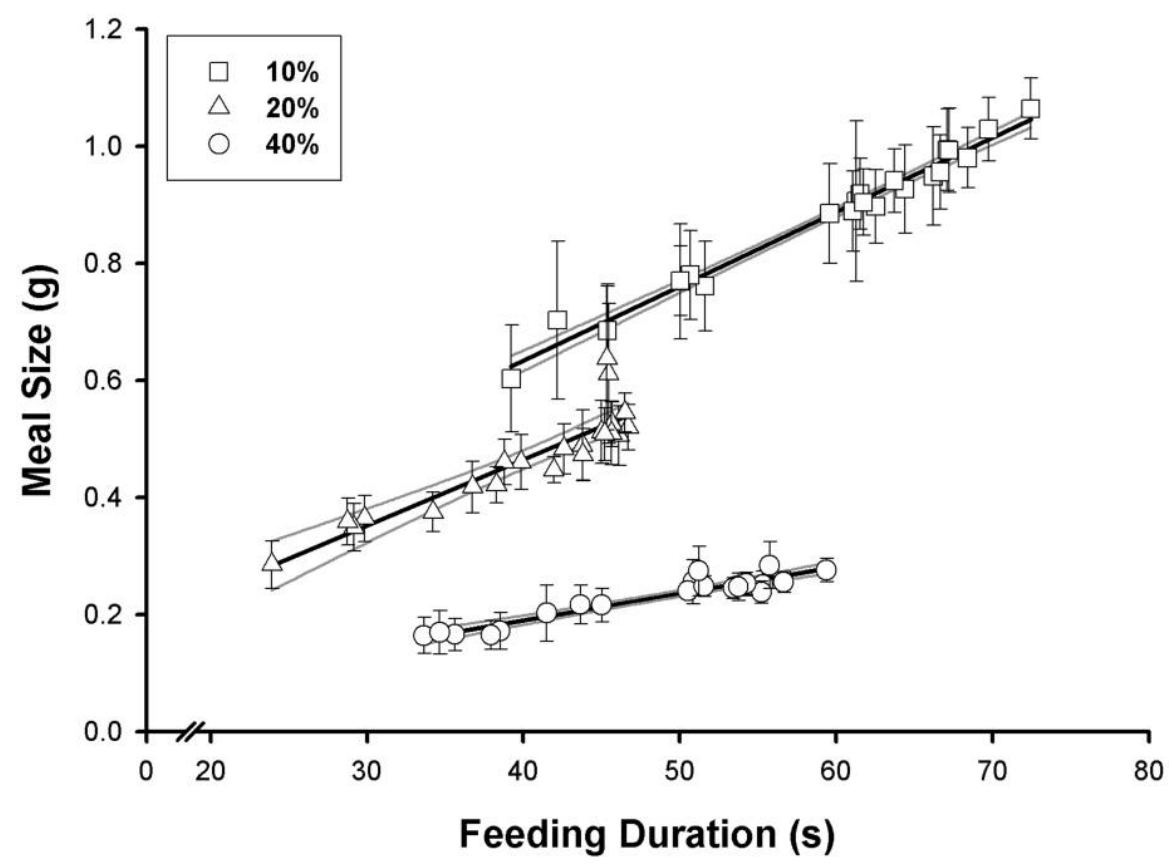

Figure 2. Meal size (g) as a function of feeding duration (s) for 10 sunbirds each fed three sucrose concentrations (10\%, 20\%, and $40 \% \mathrm{w} / \mathrm{w})$. Each data point (mean $\pm \mathrm{SE}$ ) represents one 30-min interval $(n=22$ per concentration). Gray lines represent $95 \%$ confidence intervals; $X$-axis error bars were omitted for clarity.

$0.0012] x+0.014, \quad R^{2}=0.82 ; \quad 40 \%: \quad y=0.005[ \pm 0.0003] x+$ $\left.0.008, R^{2}=0.90\right)$.

Intake rates for all 10 sunbirds, obtained from the regression analysis of feeding duration and meal size on the three diets, are shown in Figure 3. The intake rate decreased with increasing sucrose concentration $\left(F_{2,18}=27.86, P<0.001\right)$. Post hoc analysis revealed a significant difference in intake rates between $10 \%$ and $40 \%(P<0.001)$ and between $20 \%$ and $40 \%(P<$ $0.001)$. The intake rates on the $10 \%$ and $20 \%$ sucrose concentrations did not differ $(P=0.67)$.

Feeding frequency. A positive relationship was evident between meal size and feeding frequency; 24 out of the 30 linear regression analyses of all three sucrose concentrations were significant (all $F_{1,8} \geq 5.63$, all $P \leq 0.03$, all $R^{2} \geq 0.22$ ). However, the regression slopes varied greatly between individuals. Mean values of these slopes (mg per feeding event) \pm SE (range) were 10\%: $10.2 \pm 1.4$ (3.2-16.0); 20\%: $8.0 \pm 1.5$ (1.7-16.2); 40\%: $4.2 \pm 0.8(0.9-8.1)$. Therefore, our analysis focuses exclusively on the relationship between feeding duration and meal size.

\section{Differences between the Sexes and Body Mass Relationships}

A significant sexual body mass dimorphism was evident $(U<$ $0.01, P<0.01$ ), with males being $15.2 \%$ heavier than females. No significant difference between the sexes was found for feeding duration (10\%: $F_{1,8}=0.61, P=0.46 ; 20 \%: F_{1,8}=0.26$, $\left.P=0.62 ; 40 \%: F_{1,8}=0.44, P=0.53\right)$ or meal size $(10 \%$ : $F_{1,8}=4.61, P=0.06 ; 20 \%: F_{1,8}=1.17, P=0.31 ; 40 \%: F_{1,8}=$ 3.84, $P=0.09$ ). However, there appeared to be a weak rela- tionship between sex and either feeding duration or meal size, with males feeding for longer and consuming larger amounts than females on all diets. Feeding duration over the whole day was not related to body mass on any diet concentration (all $F_{1,8} \leq 0.49$, all $P \geq 0.51$, all $R^{2} \leq 0.06$ ). There was also no significant relationship between the mass of solution consumed

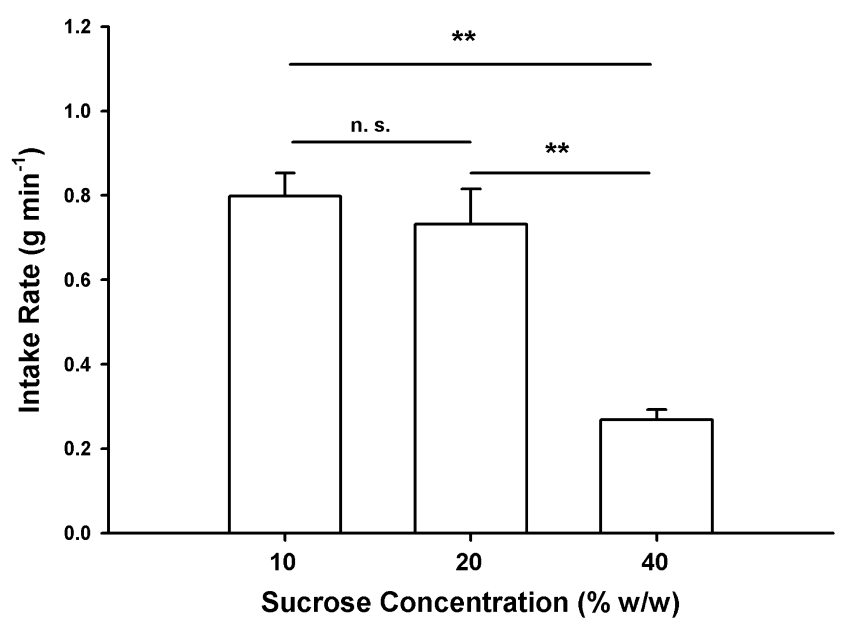

Figure 3. Intake rates for individual birds $(n=10$; mean \pm SE) for the three sucrose concentrations. The ranges in intake rate $\left(\mathrm{g} \mathrm{min}^{-1}\right)$ for each concentration were as follows: $10 \%: 0.61-1.15 ; 20 \%: 0.51-$ $1.40 ; 40 \%: 0.19-0.39$. Statistical results derive from the Tukey's honest significant difference post hoc test that followed comparison of the intake rates on the different diets by repeated-measures ANOVA (n.s. $=P>0.05$; two asterisks $=P<0.01)$. 
daily and body mass on any of the diet concentrations (all $F_{1,8} \leq 1.33$, all $P \geq 0.28$, all $R^{2} \leq 0.14$ ). Feeding frequency did not differ between the sexes $\left(10 \%: F_{1,8}=0.87, P=0.38 ; 20 \%\right.$ : $\left.F_{1,8}=2.81, P=0.13 ; 40 \%: F_{1,8}=1.80, P=0.22\right)$, but females appeared to have higher feeding frequencies than males on all diets. The intake rates on each diet concentration (Fig. 3) did not differ between the sexes $\left(F_{1,8}=0.11, P=0.75\right)$.

\section{Daily Rhythm, Feeding Patterns, and Consumption} on the Different Diets

Irrespective of diet concentration, birds showed a daily rhythm in their feeding patterns. Both feeding duration $\left(F_{2,21}=5.02\right.$, $P<0.001)$ and meal size $\left(F_{2,21}=5.37, P<0.001\right)$ varied significantly over the course of the day. Both were lower in the last $2.5 \mathrm{~h}$ of the experimental period than during the rest of the day (feeding duration: $P \leq 0.03$; meal size: $P \leq 0.003$ ). In Figure 2 , these evening intervals lie at the lower end of the regression lines for all three sucrose concentrations.

Feeding duration differed between sucrose concentrations $\left(F_{2,21}=31.86, P<0.001\right)$. Feeding duration was lower on the $20 \%$ diet than on $10 \%$ but increased on the $40 \%$ diet (Fig. 2). Post hoc analysis showed significant differences in feeding duration between all concentrations $(10 \%$ and $20 \%: P<0.001$; $10 \%$ and $40 \%: P<0.001 ; 20 \%$ and $40 \%: P=0.01)$. Meal size decreased with increasing diet concentration (Fig. 2; $F_{2,21}=$ 424.30, $P<0.001)$. Post hoc analysis revealed a significant difference in meal size between all concentrations $(P<0.001)$. Mean masses of solution consumed daily $(\mathrm{g}) \pm$ SE were $10 \%$ : $19.30 \pm 0.75 ; 20 \%$ : $10.26 \pm 0.35 ; 40 \%: 5.04 \pm 0.18\left(F_{2,18}=\right.$ $861.39, P<0.0001)$. Post hoc analysis showed a significant difference in mass of solution consumed daily between all concentrations $(P<0.001)$. The daily sucrose intake did not differ between diet concentrations $\left(F_{2,18}=2.59, P=0.08\right)$. Mean values of daily sucrose intake $(\mathrm{g}) \pm \mathrm{SE}$ were $10 \%$ : $1.93 \pm 0.08$; 20\%: $2.05 \pm 0.07 ; 40 \%: 2.02 \pm 0.07$.

\section{Discussion}

\section{Meal Size and Feeding Duration}

Feeding duration of white-bellied sunbirds has been clearly demonstrated to be an index of the meal size on a particular sugar concentration. This evidence of the positive relationship between feeding duration and meal size has major implications for other studies because the relationship could only be assumed before now. With the exception of one study on western spinebills (Acanthorhynchus superciliosis), which showed that feeding durations are positively related to volumes of nectar consumed (Collins and Clow 1978), no other evidence exists for this relationship in nectar-feeding birds. In hovering hawk moths (Macroglossum stellatarum), a relationship between feeding duration and the volume of sucrose solution ingested has also been demonstrated (Josens and Farina 2001). A study measuring the food intake of Sprague-Dawley rats (Rattus norvegicus), however, showed that the correlation between meal size and feeding duration is not as strong as would be needed to accurately estimate meal size from duration data (Castonguay et al. 1986). Cameron (1998) reviewed studies that have correlated time spent suckling by mammalian infants with their milk intake. Less than half of the reviewed studies found a significant positive relationship, which is insufficient evidence that time spent suckling provides an index of milk intake (Cameron 1998).

\section{Viscosity Effects and Compensatory Feeding}

The intake rate depended on the sugar concentration of the diet, with the highest rate at the lowest concentration of $10 \%$ and the lowest rate at the $40 \%$ concentration. Increasing viscosity of the solution with increasing concentration might explain this observation. A study on licking rates of rufous hummingbirds (Selasphorus rufus) showed that both licking frequency and volume per lick decreased with increasing sugar concentration (Roberts 1995). In our study, feeding duration on the $40 \%$ solution was prolonged because of the increased viscosity, accentuated by the Ensure, which was added in adjusted quantity to the experimental diets (see "Material and Methods"). As a result, feeding duration was lower for the $20 \%$ diet than for the $10 \%$ diet but was found to increase on the $40 \%$ diet, although meal size decreased with increasing sugar concentration because of compensatory feeding (Nicolson and Fleming 2003). Thus, birds were able to maintain a constant daily energy intake by adjusting meal size according to diet concentration.

\section{Daily Rhythm in Feeding Patterns}

Irrespective of diet concentration, birds showed a daily rhythm in their feeding patterns. Both feeding duration and meal size were found to be lower in the late afternoon and evening compared with the rest of the day. Our previous study also showed the tendency for birds to spend less time feeding during the afternoon than in the late morning (Köhler et al. 2006). A rather different pattern was evident when food intake was examined on an hourly basis: after feeding steadily during the morning, white-bellied sunbirds reduced their intake during the afternoon, and food intake increased at the end of the day to provide energy stores for the night (Fleming et al. 2004).

\section{Differences between the Sexes and Individual Variation}

A sexual body mass dimorphism was evident for white-bellied sunbirds used in this study, which suggests possible differences in food intake between the sexes. Markman et al. (2006) demonstrated sex-specific differences in the transit time of Palestine sunbirds (Cinnyris oseus), which might affect foraging behavior. However, the sexual-size dimorphism of their birds was exceptionally high, with males being $28 \%$ heavier than females (Markman et al. 2006). In our study, the sexes did not differ in their feeding patterns, despite males being 15\% heavier than 
females. However, males appeared to feed for longer and have larger meal sizes than females on all diets. This may indicate the possibility of a sex-specific feeding pattern because there was no sex-independent relationship between body mass and both feeding duration over a whole day and mass of solution consumed daily. Interestingly, females appeared to have higher feeding frequencies than males. Females' meal size might be smaller because of their smaller body size (and thus greater volumetric constraint because sunbirds do not have a crop), and they might compensate by feeding more often.

Sex-specific feeding patterns were probably obscured by individual feeding patterns. Interindividual differences were also evident in the large variation in the regression slopes obtained from the relationship between meal size and feeding frequency. Our previous work has already highlighted pronounced variation between individual sunbirds, which differ greatly in their feeding frequency, and the duration of feeding events (Köhler et al. 2006). Nicolson and Fleming (2003) also demonstrated large variation in sucrose intake between individual whitebellied sunbirds. Therefore, we suggest that the number of birds used in further investigations could be increased to avoid the likelihood of sex-specific feeding patterns being obscured by interindividual variation.

\section{Acknowledgments}

This project was funded by the South African National Research Foundation. We are grateful to Jan Cilliers Park for permission to mist-net sunbirds under permit from the Gauteng Directorate of Nature Conservation. Our experiments were approved by the Animal Use and Care Committee of the University of Pretoria.

\section{Literature Cited}

Blanton C.A., B.A. Horwitz, C. Murtagh-Mark, D.W. Gietzen, S.M. Griffey, and R.B. McDonald. 1998. Meal patterns associated with the age-related decline in food intake in the Fischer 344 rat. Am J Physiol 275:R1494-R1502.

Cameron E.Z. 1998. Is suckling behaviour a useful predictor of milk intake? a review. Anim Behav 56:521-532.

Castonguay T.W., L.L. Kaiser, and J.S. Stern. 1986. Meal pattern analysis: artefacts, assumptions and implications. Brain Res Bull 17:439-443.

Collins B.G. and H. Clow. 1978. Feeding behaviour and energetics of the western spinebill, Acanthorhynchus superciliosis (Aves: Meliphagidae). Aust J Zool 26:269-277.

Fleming P.A., D.A. Gray, and S.W. Nicolson. 2004. Circadian rhythm of water balance and aldosterone excretion in the whitebellied sunbird Nectarinia talatala. J Comp Physiol B 174:341-346.
Garrison J.S.E. and C.L. Gass. 1999. Response of a traplining hummingbird to changes in nectar availability. Behav Ecol 10:714-725.

Gass C.L. 1978. Experimental studies of foraging in complex laboratory environments. Am Zool 18:729-738.

Hainsworth F.R., E. Precup, and T. Hamill. 1991. Feeding, energy processing rates and egg production in painted lady butterflies. J Exp Biol 156:249-265.

Jackson S., S.W. Nicolson, and C.N. Lotz. 1998. Sugar preferences and "side bias" in Cape sugarbirds and lesser doublecollared sunbirds. Auk 115:156-165.

Josens R.B. and W.M. Farina. 2001. Nectar feeding by the hovering hawk moth Macroglossum stellatarum: intake rate as a function of viscosity and concentration of sucrose solutions. J Comp Physiol A 187:661-665.

Kahler A., N. Geary, L.A. Eckel, L.A. Campfield, F.J. Smith, and W. Langhans. 1998. Chronic administration of OB protein decreases food intake by selectively reducing meal size in male rats. Am J Physiol 275:R180-R185.

Köhler A., L. Verburgt, and S.W. Nicolson. 2006. Short-term energy regulation of whitebellied sunbirds (Nectarinia talatala): effects of food concentration on feeding frequency and duration. J Exp Biol 209:2880-2887.

López-Calleja M.V. and F. Bozinovic. 2003. Dynamic energy and time budgets in hummingbirds: a study in Sephanoides sephaniodes. Comp Biochem Physiol A 134:283-295.

Markman S., H. Tadmor-Melamed, A. Arieli, and I. Izhaki. 2006. Sex differences in food intake and digestive constraints in a nectarivorous bird. J Exp Biol 209:1058-1063.

Mitchell R.J. and D.C. Paton. 1990. Effects of nectar volume and concentration on sugar intake rates of Australian honeyeaters (Meliphagidae). Oecologia 83:238-246.

Nicolson S.W. and P.A. Fleming. 2003. Energy balance in the whitebellied sunbird Nectarinia talatala: constraints on compensatory feeding, and consumption of supplementary water. Funct Ecol 17:3-9.

Nicolson S.W., D. Hoffmann, and P.A. Fleming. 2005. Shortterm energy regulation in nectar-feeding birds: the response of whitebellied sunbirds (Nectarinia talatala) to a midday fast. Funct Ecol 19:988-994.

Paul J. and F. Roces. 2003. Fluid intake rates in ants correlate with their feeding habits. J Insect Physiol 49:347-357.

Rice W.R. 1989. Analyzing tables of statistical tests. Evolution 43:223-225.

Roberts W.M. 1995. Hummingbird licking behaviour and the energetics of nectar feeding. Auk 112:456-463.

Van Tets I.G. and S.W. Nicolson. 2000. Pollen and the nitrogen requirements of the lesser double-collared sunbird. Auk 117: 826-830.

Zar J.H. 1999. Biostatistical Analysis. 4th ed. Prentice Hall, Upper Saddle River, NJ. 